\title{
A Rapid Culture Technique Produces Functional Dendritic-Like Cells from Human Acute Myeloid Leukemia Cell Lines
}

\author{
Jian Ning, ${ }^{1,2}$ David Morgan, ${ }^{2}$ and Derwood Pamphilon ${ }^{1,2}$ \\ ${ }^{1}$ Immunotherapy Laboratory, Stem Cell R\&D, Bristol Institute for Transfusion Sciences, NHS Blood and Transplant, North Bristol Park, \\ Northway, Filton, Bristol BS34 7QH, UK \\ ${ }^{2}$ School of Cellular and Molecular Medicine, School of Medical Sciences, University of Bristol, University Walk, Bristol BS8 1TD, UK
}

Correspondence should be addressed to David Morgan, d.j.morgan@bristol.ac.uk

Received 20 June 2011; Revised 4 August 2011; Accepted 18 August 2011

Academic Editor: Margherita Gigante

Copyright () 2011 Jian Ning et al. This is an open access article distributed under the Creative Commons Attribution License, which permits unrestricted use, distribution, and reproduction in any medium, provided the original work is properly cited.

\begin{abstract}
Most anti-cancer immunotherapeutic strategies involving dendritic cells (DC) as vaccines rely upon the adoptive transfer of DC loaded with exogenous tumour-peptides. This study utilized human acute myeloid leukemia (AML) cells as progenitors from which functional dendritic-like antigen presenting cells (DLC) were generated, that constitutively express tumour antigens for recognition by $\mathrm{CD} 8^{+} \mathrm{T}$ cells. DLC were generated from AML cell lines KG-1 and MUTZ-3 using rapid culture techniques and appropriate cytokines. DLC were evaluated for their cell-surface phenotype, antigen uptake and ability to stimulate allogeneic responder cell proliferation, and production of IFN- $\gamma$; compared with DC derived from normal human PBMC donors. KG-1 and MUTZ-3 DLC increased expression of CD80, CD83, CD86, and HLA-DR, and MUTZ-3 DLC downregulated CD14 and expressed CD1a. Importantly, both KG-1 and MUTZ-3-derived DLC promoted proliferation of allogeneic responder cells more efficiently than unmodified cells; neither cells incorporated FITC-labeled dextran, but both stimulated IFN- $\gamma$ production from responding allogeneic $\mathrm{CD}^{+} \mathrm{T}$ cells. Control DC produced from PBMC using the FastDC culture also expressed high levels of critical cell surface ligands and demonstrated good APC function. This paper indicates that functional DLC can be cultured from the AML cell lines KG-1 and MUTZ-3, and FastDC culture generates functional KG-1 DLC.
\end{abstract}

\section{Introduction}

Manipulating the interactions between DC and naïve T cells is an important part of the design of strategies for the immunotherapy of both solid and hematological cancers [1]. In vitro, $\mathrm{DC}$ can be generated from $\mathrm{CD} 34^{+}$cells by culture with cytokines such as GM-CSF, IL-4, Flt3L, CD40 ligand, SCF, and TGF- $\beta$. Alternatively DC can be grown from CD $14^{+}$ peripheral blood mononuclear cells (PBMC) during 5-9day culture, using GM-CSF and IL-4 [2]. DC maturation is usually achieved with either TNF- $\alpha$ or lipopolysaccharide (LPS). It was also reported that functional DC could be cultured from PBMC using a combination of GM-CSF and IL-4 for 24 hours followed by a combination of four proinflammatory cytokines (TNF- $\alpha$, IL- $1 \beta$, IL-6, and PGE 2 ) for a further 24 hours [3].

We and others have previously reported that DC can be cultured from leukemic blast cells [4-6]. In some cases either autologous $[6,7]$ or allogeneic $[5,6]$ antileukemic cytotoxic T lymphocytes (CTL) were generated. Alternatively, normal DC are pulsed with leukemia antigens using either apoptotic blasts or leukemic cell lysate, or DC-tumor cell hybrids are used [1]. In addition, some available human leukemic cell lines can be differentiated into DLC [8]. In this study we have utilized AML cell lines for use as a source of DLC. Importantly, this approach provides a uniform source of DLC for further study.

Long-term DC culture protocols may increase experimental cost and the possibility of contamination, and optimized culture protocols have been investigated to evaluate the utility of shorter cultures which may minimize microbiological contamination and be more economical. Therefore, the culture of unselected normal PBMC using the FastDC method described by Dauer et al. [3], and "conventional" 7-day DC culture with GM-CSF and IL-4 were compared. Experiments were also carried out using 
optimized methods for the differentiation of DLC from the human leukemia cell lines KG-1 and MUTZ-3, which have myeloid and monocytic characteristics, respectively. In this study, the term DLC has been restricted to the cells obtained from leukemic cell line cells after DC culture. Previous reports have shown that GM-CSF, IL-4, PMA, and ionomycin were able to induce the formation of DLC from KG-1 cells [9-11] whereas MUTZ-3 DLC are best cultured using the more conventional combination of GMCSF, IL- 4 , and TNF- $\alpha$ over 5 days $[12,13]$. Studying DLC function could be vital to understand the biology of DC and aid the development of immunotherapeutic strategies to combat cancer. DLC transformation of cultured KG-1 and MUTZ-3 cells was studied using FastDC and 7-day culture methods. The cell-surface phenotypes of DC and DLC were evaluated together with functional analysis of FITCconjugated dextran phagocytosis, stimulation of allogeneic T-cell proliferation, and IFN- $\gamma$ production.

\section{Materials and Methods}

2.1. Antibodies and Reagents. FITC-labeled anti-human CD1a(HI149), CD80(L307.4), CD83(HB15e), HLA-ABC (G46-2.6), HLA-DR(G46-6), and PE-labeled anti-human CD11c(B-ly6), CDw123(9F5), CD14(M5E2), CD40(5C3), CD54(HA58), CD8(HIT8a), and CD86(IT2.2) antibodies were obtained from BD PharMingen (Cowley, UK). FITCand PE-labeled isotype control antibodies $\mathrm{IgG}_{1} \kappa$ were obtained from BD PharMingen. FITC-labeled dextran (molecular weight $40 \mathrm{kDa}$ ) was purchased from Sigma-Aldrich (Poole, UK). APC-labeled anti-human IFN- $\gamma$ antibody was purchased from eBioscience (Hatfield, UK). Recombinant human GM-CSF, IL- $1 \beta$, IL-4, IL-6, TGF- $\beta$, and TNF- $\alpha$ were obtained from R\&D Systems (Abingdon, UK). Ionomycin, PGE2 and phorbol 12-myristate 13-acetate (PMA) were purchased from Sigma-Aldrich.

2.2. Peripheral Blood Samples. PBMC were obtained using blood samples taken from healthy apheresis donors with informed consent, and the Central and South Bristol Research Ethics Committee approved the study.

2.3. Cell Line Culture. The KG-1 human AML cell line was purchased from ECACC (Salisbury, UK). The cytokinedependent MUTZ-3 acute myelomonocytic leukaemia and 5637 cell lines were obtained from DSMZ (Braunschweig, Germany). KG-1 was cultured in Iscove's modified Dulbecco's medium with GlutaMAX I (IMDM, GibcoInvitrogen, Paisley, UK) plus 20\% (v/v) fetal bovine serum (FBS, Sigma-Aldrich). MUTZ-3 was maintained in minimum essential medium- $\alpha$ (Gibco-Invitrogen) plus $20 \%$ $(\mathrm{v} / \mathrm{v}) \mathrm{FBS}$ and $10 \%$ conditioned medium prepared from the 5637 bladder carcinoma cell line [14], which was cultured in IMDM supplemented with 10\% FBS (v/v). The 5637conditioned medium was collected, filtered with a $0.2 \mu \mathrm{m}$ syringe filter and kept at $-80^{\circ} \mathrm{C}$ for further use. All media were supplemented with $100 \mathrm{U} / \mathrm{mL}$ penicillin and $100 \mu \mathrm{g} / \mathrm{mL}$ streptomycin. The KG-1 and MUTZ-3 cells were passaged routinely and grown in a humidified incubator at $37^{\circ} \mathrm{C}$ with $5 \%(\mathrm{v} / \mathrm{v}) \mathrm{CO}_{2}$. HLA typing of KG-1 and MUTZ-3 cells was done by Department of Histocompatibility and Immunogenetics, NHS Blood and Transplant. HLA type of KG-1 cells is A30, B53, B78, Cw16, Cw04, DR11, DR14, and DQ06, and the MUTZ-3 HLA type is A02, A03, B44, Cw04, Cw07 and DR10, DR11, DQ03, DQ05.

2.4. Generation of DLC and DC. For generation of DLC, either KG-1 or MUTZ-3 cells were seeded at $2 \times 10^{5} /$ well in 24-well plates in IMDM containing 20\% (v/v) BIT (StemCell Technologies, Vancouver, Canada). PBMC were prepared by Ficoll (Sigma-Aldrich) separation, suspended at either $10^{6}$ cells $/ \mathrm{mL}$ or $5 \times 10^{5}$ cells $/ \mathrm{mL}$, respectively, in IMDM containing 20\% (v/v) BIT and incubated for 3 hours on 2\% $(\mathrm{v} / \mathrm{v})$ human albumin precoated 6-well plates. Suspended cells were then removed by replacing with fresh IMDM supplemented with $20 \%$ BIT (v/v) and cytokines. DLC or DC from KG-1, MUTZ-3, and PBMC were induced using two culture methods: (i) FastDC method: $20 \mathrm{ng} / \mathrm{mL}$ IL4 and $62.5 \mathrm{ng} / \mathrm{mL}$ GM-CSF for 24 hours then maturation with $9 \mathrm{ng} / \mathrm{mL}$ TNF- $\alpha, 10 \mathrm{ng} / \mathrm{mL}$ IL- $1 \beta, 10 \mathrm{ng} / \mathrm{mL}$ IL-6, and $350 \mathrm{ng} / \mathrm{mL} \mathrm{PGE} 2$ for another 24 hours [3] and for KG-1 DLC, cells were cultured for $24 \mathrm{~h}$ with all FastDC cytokines and $200 \mathrm{ng} / \mathrm{mL}$ ionomycin; (ii) 7-day culture method, where cells were stimulated with $20 \mathrm{ng} / \mathrm{mL}$ IL-4, and $50 \mathrm{ng} / \mathrm{mL}$ GM-CSF for 5 days then with $12 \mathrm{ng} / \mathrm{mL}$ TNF- $\alpha$ for 2 days for maturation. During the culture, half the volume of medium was replaced with fresh medium supplemented with cytokines every other day. The cells were cultured in a humidified incubator at $37^{\circ} \mathrm{C}$ supplied with $5 \%(\mathrm{v} / \mathrm{v}) \mathrm{CO}_{2}$.

2.5. Analyses by Flow Cytometry. The phenotypes of PBMC and derived DC were monitored using a Coulter EPICS XL flow cytometer. Further data analysis was carried out using Expo32 ADC software. The phenotypes of KG-1, MUTZ-3, and their induced DLC as well as IFN- $\gamma$ production were analyzed using BD Calibur and data were processed using WinMDI 2.9 software.

2.6. FITC-Dextran Uptake. Each cell sample was incubated in the dark with FITC-dextran $(1 \mathrm{mg} / \mathrm{mL})$ prepared with HBSS either at $37^{\circ} \mathrm{C}$ or at $4^{\circ} \mathrm{C}$ as control for $1 \mathrm{hr}$ and were then washed with cold HBSS before being analyzed by flow cytometry. Results were expressed as the ratio of mean fluorescence intensity of each sample to its control culture at $4^{\circ} \mathrm{C}$.

2.7. Mixed Lymphocyte Culture (MLC) Assay. All cells were washed once and resuspended in RPMI 1640 with $10 \%(\mathrm{v} / \mathrm{v})$ heat inactivated human AB serum (Sigma-Aldrich). MLCs were set up in 96-well round-bottom plates by culturing 50,000 cells/well responder PBMC from HLA mismatched donors with either 25 Gy ${ }^{137} \mathrm{Cs} \gamma$-irradiated allogeneic PBMC and DC, 60 Gy irradiated KG-1 and derived DLC or 80 Gy irradiated MUTZ-3 and derived DLC. The final volume of each well was $200 \mu \mathrm{L}$, and all determinations were performed in quadruplicate. Cell proliferation was assessed on day 5 

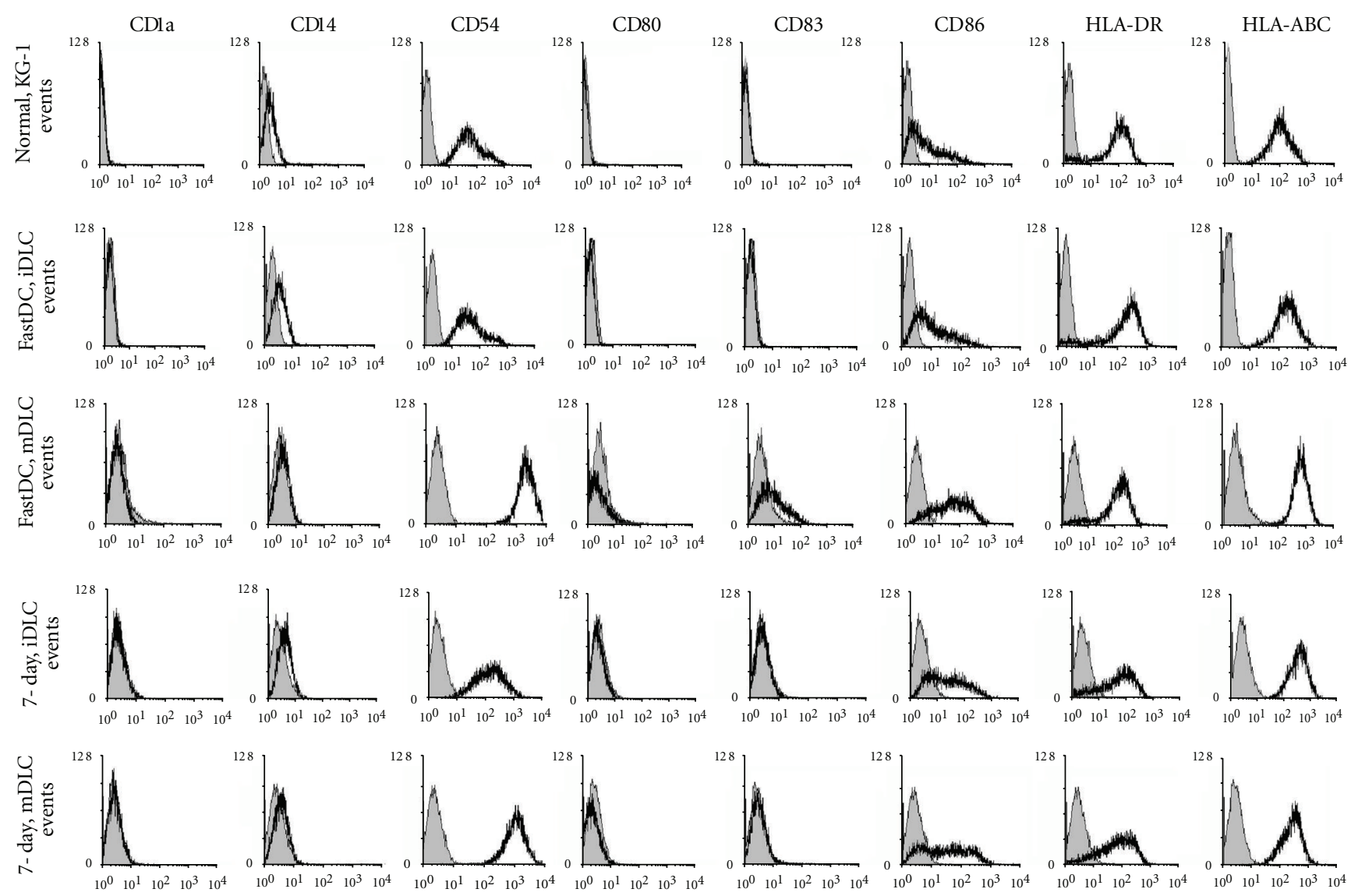

FIGURE 1: DLC phenotypes of KG-1 cells. KG-1 cells were culture to generate DLC using FastDC and 7-day culture methods. The phenotypes were monitored at immature (iDLC) and mature (mDLC) stages in each culture. Data are representative of at least three samples. Shaded histograms are isotype controls and unshaded histograms represent marker expression.

TABLE 1: Cell phenotypes of DC generated from PBMC using FastDC and 7-day culture methods. The results were the percentages of positive stained cells in gated cells after subtraction of the appropriate values of isotype controls. Results are shown as mean \pm SD, where $n$ is the total sample number tested.

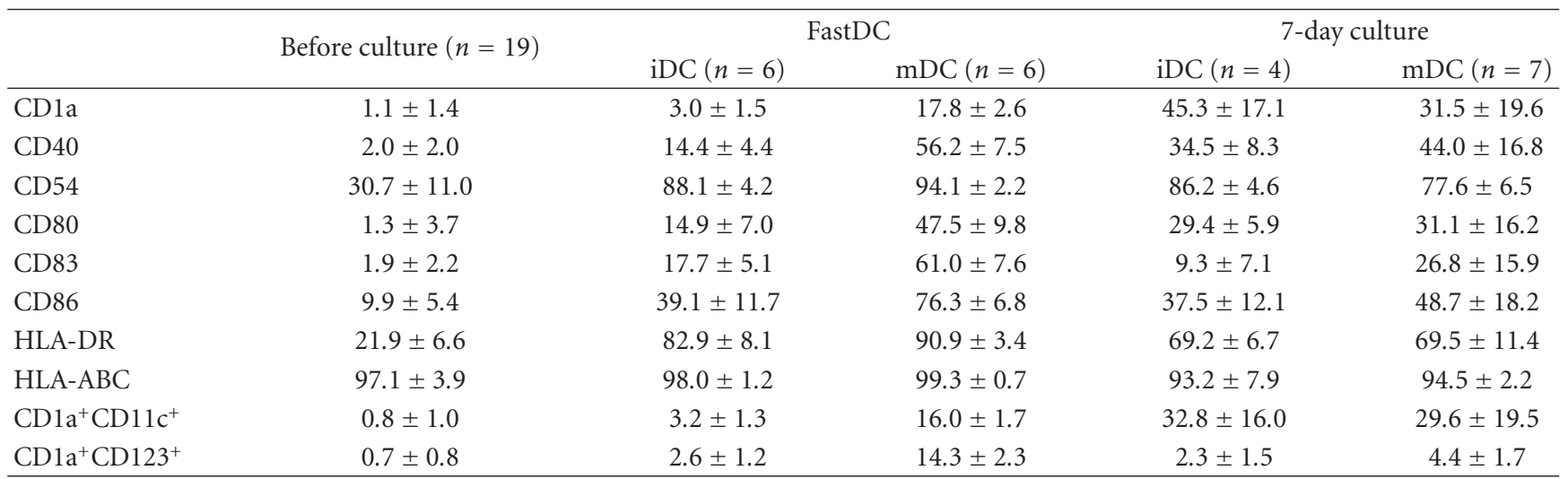

for KG-1 or day 7 for MUTZ-3 and derived DLC. DC produced from PBMC FastDC culture were used for an MLR course study from day 4 to $7.4 \mu \mathrm{Ci} /$ well of ${ }^{3} \mathrm{H}$ thymidine (GE Healthcare, Chalfont, Bucks, UK) were added into culture at the last 18 hours. Cells were harvested onto filters using a Tomtec harvester and then counted on a Wallac Tailux 1450 microBeta counter. MLC results were calculated after subtraction of the respective reading of ${ }^{3} \mathrm{H}$-thymidine incorporated into the irradiated stimulator cells alone.

2.8. In Vitro IFN- $\gamma$ Assay. In order to be distinguished from stimulator cells, responder PBMC from HLA mismatched donors were incubated with $5 \mu \mathrm{M} \mathrm{CFSE}$ at $37^{\circ} \mathrm{C}$ for $20 \mathrm{~min}$. 

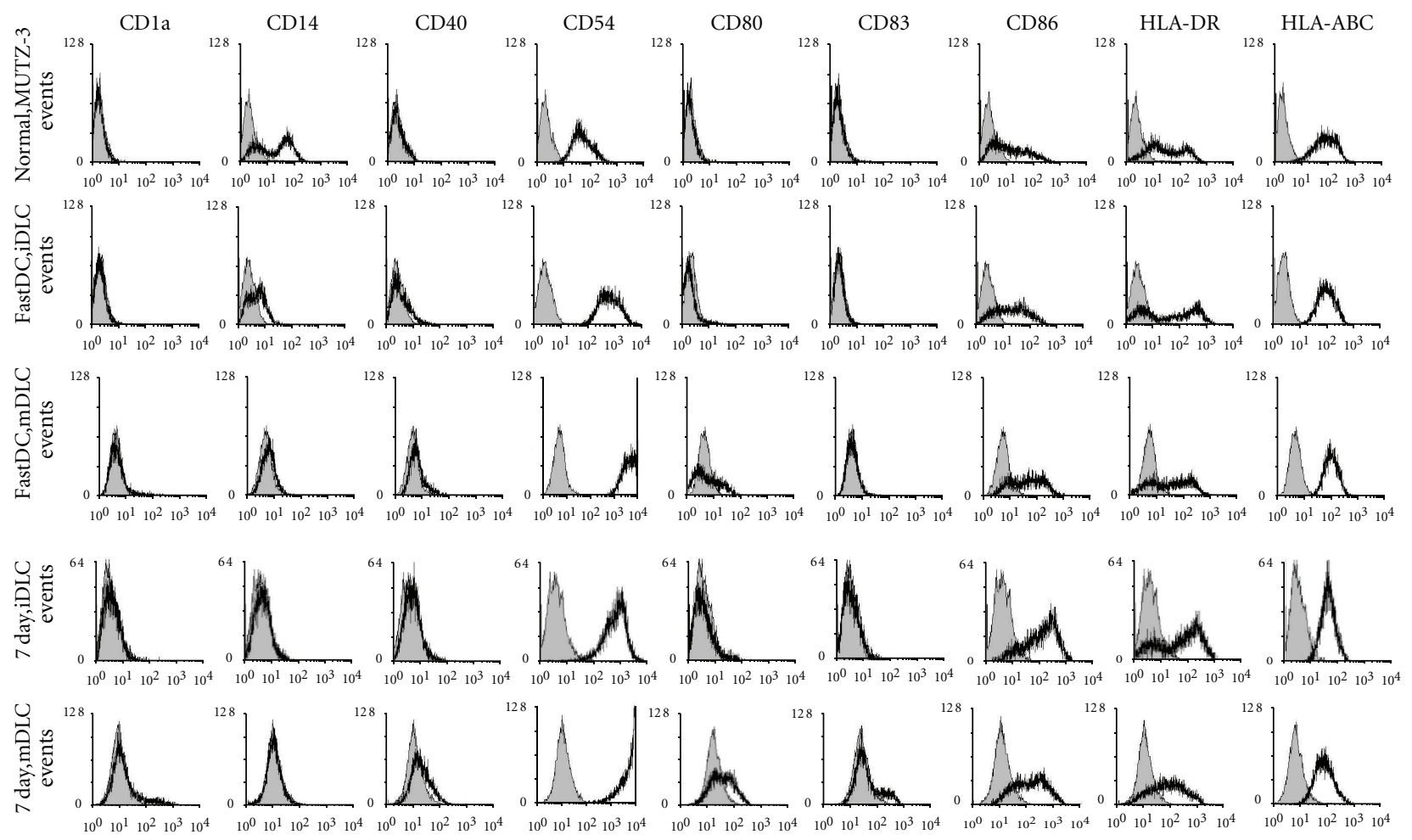

Figure 2: DLC phenotypes of MUTZ-3. DLC generated from MUTZ-3 after culture using FastDC and 7-day culture methods. One representative result from three experiments is shown. Isotype controls are shaded and surface markers are unshaded in histogram.

Irradiated KG-1, MUTZ-3, and their derived DLC and DC generated from PBMC FastDC and 7-day cultures were mixed with $5 \times 10^{4}$ responder PBMC at the ratio of $1: 10$ in each well in round-bottom 96-well plates. The plates were cultured for 4 days and $10 \mathrm{ng} / \mathrm{mL}$ PMA and $200 \mathrm{ng} / \mathrm{mL}$ ionomycin were added into the culture for the last $16 \mathrm{hrs}$. On the day of harvest, GolgiPlug (BD Biosciences) was added to each well and incubated for 3 hours. The cells were stained with CD8-PE antibody then resuspended in BD Cytofix/Cytoperm buffer to permeabilise for $20 \mathrm{~min}$ at $4^{\circ} \mathrm{C}$. After washing with BD Perm/wash buffer, the cells were stained with IFN- $\gamma$-APC antibody before analysis by flow cytometry.

2.9. Statistical Analysis. Statistical analysis was carried out using one-way ANOVA followed by Dunnett's test.

\section{Results}

3.1. DLC Phenotype Using Cultured KG-1 and MUTZ-3 Cells. Normal cultured KG-1 cells expressed high levels of CD54, HLA-DR, and HLA-ABC and low levels of CD86, but not CD1a, CD80, and CD83 (Figure 1). After culture using the FastDC method, de novo expression of CD83 and upregulation of CD86 and CD54 were observed at the maturation stage. In the 7-day cultures, CD54 expression was increased but no CD83 expression was observed. Moderate levels of expression of HLA-DR and a high level of HLA$\mathrm{ABC}$ were seen in both induction methods. Importantly the
DLC generated were not monocytes as confirmed by the lack of CD14 expression on their surface. Although there were only minor differences between the phenotypes of the DCs generated using FastDC and 7-day culture techniques, we selected the FastDC method as this has been most frequently described in the literature [15].

MUTZ-3 cells were also tested for their ability to differentiate and display a DLC phenotype (Figure 2). After maturation in both the FastDC and 7-day cultures, the expression of CD14 observed on normal MUTZ-3 surface disappeared. DLC derived from MUTZ-3 upregulated expression of CD54 and CD86 while HLA-DR and HLA-ABC expression remained constant throughout. Comparing the two induction methods, there were higher expression levels of CD1a, CD40, CD80, and CD83 in the 7-day culture. From these results the 7-day culture method was chosen to generate DLC from MUTZ-3 for functional experiments.

3.2. Phenotypic Characterization of DC Differentiated from $P B M C$. PBMC from healthy blood donors were stimulated using either FastDC or 7-day culture methods (Figure 3 and Table 1). Before culture, PBMC expressed low levels of CD1a, CD80, and CD83 and low levels of CD86 and HLA-DR whereas after culture, expression of CD1a, CD80, and CD83 was increased on the iDC and were further elevated after maturation of the DC. Improved levels of CD54, CD86, and HLA-DR and high expression of MHC I was also observed using both methods for both $\mathrm{iDC}$ and $\mathrm{mDC}$ (Table 1). The highest levels of CD80, CD83, CD86, and HLA-DR 

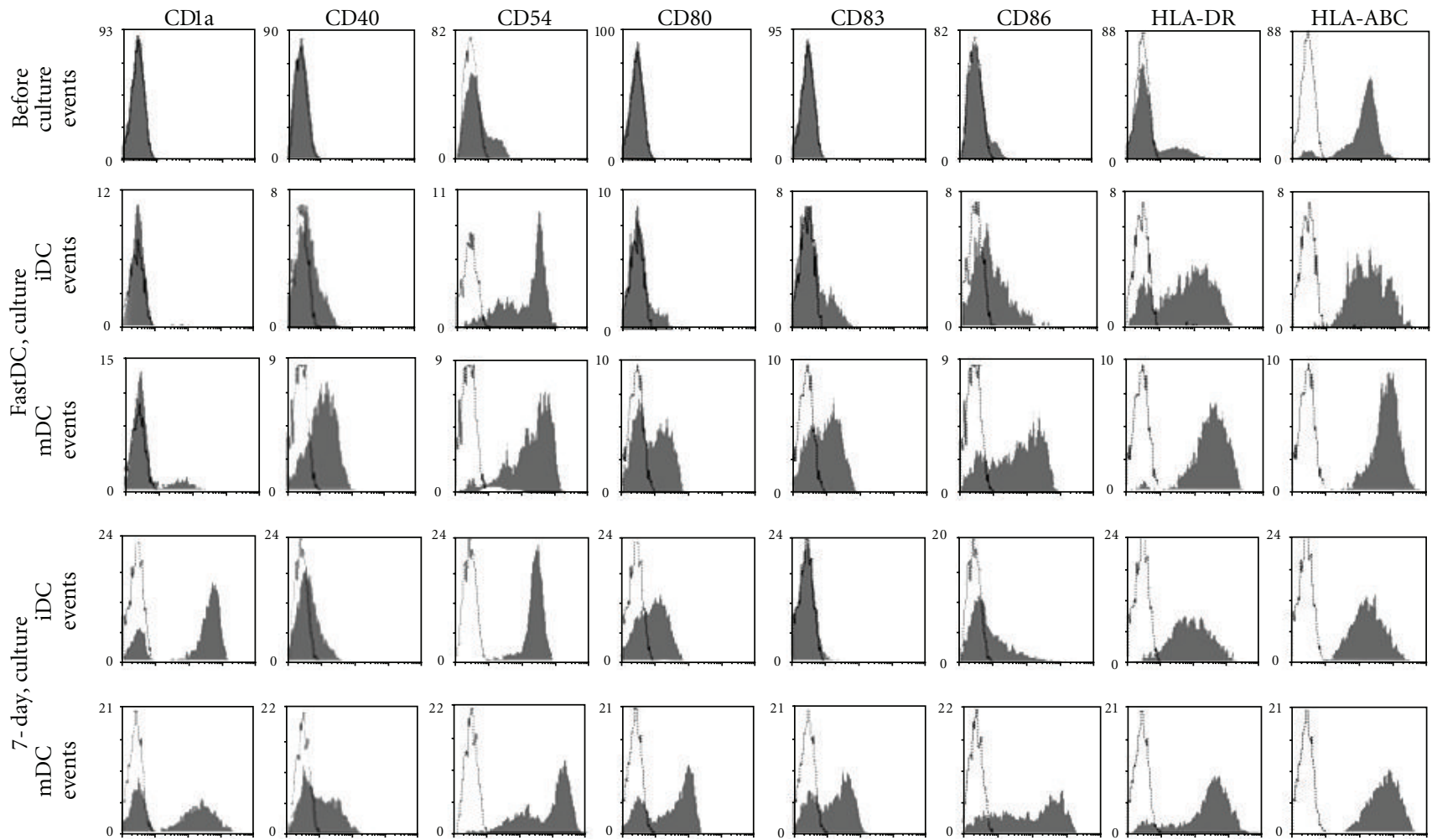

FIGURE 3: Flow cytometric analyses of iDC and mDC generated from PBMC of the same donor using the FastDC and 7-day culture methods. PBMC freshly prepared from one donor were analysed before culture and then were cultured for DC generation using both the FastDC and 7 -day methods. In FastDC culture cells were cultured in IL- 4 and GM-CSF for first 24 hrs followed by another 24 hrs to mature DC in TNF- $\alpha$, IL-1 $\beta$, IL-6, and PGE 2 . In 7-day culture, PBMC were stimulated in IL-4 and GM-CSF at iDC stage for 5 days then with TNF- $\alpha$ for 2 days to obtain mDC. Data are representative of at least three samples. Unshaded histograms drawn with broken lines are isotype controls and shaded histograms represent marker expression.

expression were observed amongst $\mathrm{mDC}$ after 2-day culture. Differentiation into myeloid DC (myDC) and plasmacytoid DC (pDC) subtypes was defined using $\mathrm{CD} 1 \mathrm{a}^{+} \mathrm{CD} 11 \mathrm{c}^{+}$and $\mathrm{CD} 1 \mathrm{a}^{+} \mathrm{CD} 123^{+}$, respectively, to delineate the two subsets. In FastDC culture, percentages of both myDC and pDC increased during the culture period and reached a similar level at mature stage. In 7-day culture there were more myDC than $\mathrm{pDC}$ at the end of culture (Table 1). In addition it was found that the cell output number was highest in PBMC FastDC culture (data not shown).

3.3. Dextran-FITC Uptake. Endocytosis is a functional feature of iDC, which decreases with maturation. iDC from PBMC demonstrated high endocytotic capability in both FastDC and 7-day culture with ratios $>2$ compared to $4^{\circ} \mathrm{C}$ control for each measurement (Figure 4). However, DLC derived from KG-1 or MUTZ-3 showed only minimal increase in Dextran-FITC uptake (Figure 4).

3.4. MLC Experiments Using DLC and DC Stimulator Cells. DLC derived from either KG-1 or MUTZ-3 and their unmodified cells were used as stimulators after irradiation to inhibit their growth (see above) although some proliferation could still be detected after irradiation, particularly in the first 3 days of culture (data not shown). Unmanipulated KG-1 cells were able to cause moderate PBMC stimulation (Figure 5(a)); as illustrated by the fact that proliferation of responder cells $(>10,000 \mathrm{cpm})$ was only observed at high stimulator-to-responder ratios $(1: 1,1: 10)$. In contrast, DLC produced from KG-1 cells showed enhanced ability to stimulate allogeneic PBMC responder cells. Likewise, MUTZ-3 DLC but not unmodified MUTZ-3 cells stimulated allogeneic T-cell proliferation in MLR (Figure 5(b)). Allogeneic DC produced from FastDC culture significantly increased the proliferation of PBMC (Figure 6(a)). The ability of generated DC to cause T-cell proliferation was superior to that of PBMC. The proliferation of PBMC after coculture with allogeneic DC from FastDC cultures was measured between day 4 and day 7 and was maximal on day 7 (Figure 6(b)).

3.5. IFN- $\gamma$ Production from Allogeneic T Cells Stimulated with DLC. When KG-1 and MUTZ-3 DLC were cocultured with allogeneic T cells, they stimulated production of IFN- $\gamma$ which was greater than that seen with unmodified KG-1 and MUTZ-3 cells, (Figure 7). The increase in IFN- $\gamma$ staining was more marked for MUTZ-3 DLC. In addition, DC obtained from FastDC and 7-day cultures stimulated responder $\mathrm{CD}^{+}$ T cell IFN- $\gamma$ production to similar levels. In the culture of 


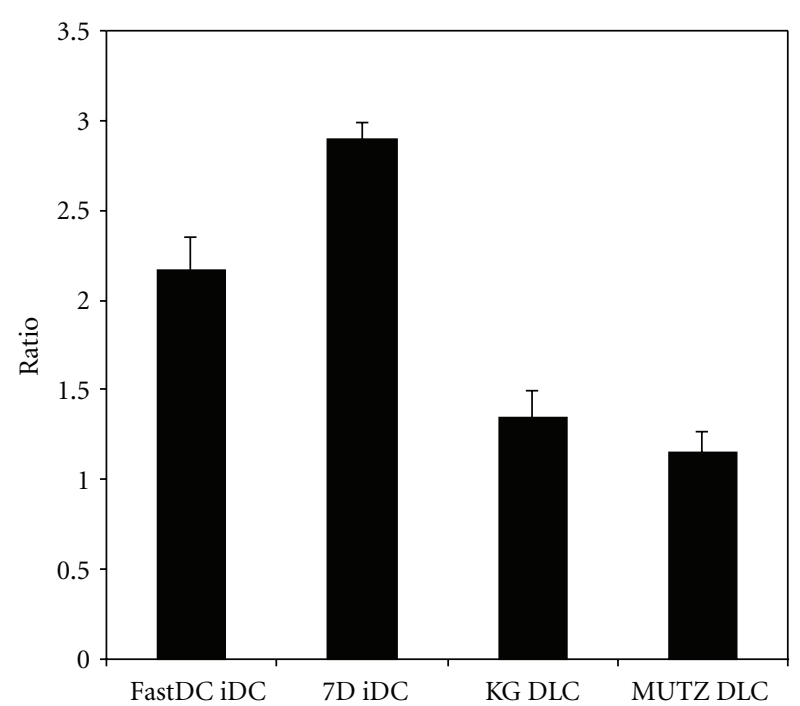

FIgURe 4: Dextran uptake of immature DC from PBMC and DLC differentiated from KG-1 and MUTZ-3 cells. Dextran uptake of each sample was detected by flow cytometry after $1 \mathrm{hr}$ of incubation at $37^{\circ} \mathrm{C}$ or on ice and MFI ratio is presented. Tests were carried out in PBMC on day 1 of FastDC (FastDC iDC) and day 5 of 7-day culture (7D iDC), as well as differentiated DLC from KG-1 (KD DLC) and MUTZ-3 cells (MUTZ DLC) via FastDC culture and 7day culture, respectively. Results are shown as mean \pm SEM, at least three samples were tested.

responder alone, after PMA and ionomycin stimulation for 16 hrs, IFN- $\gamma$ level was only about $1 \%$ of gated $\mathrm{CD}^{+} \mathrm{T}$ cells (data not shown).

\section{Discussion}

The use of DLC derived from leukemic cells to stimulate tumor antigen-specific T-cell responses is of considerable interest. Investigation of CTL responses to leukemic cells may be hampered by lack of material, and the use of leukemic cell lines may allow a greater number of experiments to be performed, although selection of HLA-matched donors may be problematic with leukemia cell lines of unusual HLA type. The potential of AML cells to develop into DLC may be determined by their stage of lineage commitment and therefore this may be related to the clinical classification of the leukemia from which they are derived [16]. Previous reports have shown varying degrees of DLC development from the KG-1 and THP-1 cell lines which show myeloid and monocytic lineage commitment, respectively $[9,16]$. The myelomonocytic cell line, U937 fails to readily produce DLC probably because it has progressed too far along the macrophage differentiation pathway and undifferentiated leukemias are also of limited utility, producing less than $10 \%$ DLC [16]. Reports indicate that the MUTZ-3 cell line which shows intermediate differentiation is readily able to undergo DLC differentiation [17]. The results of this study concur with other reports and show that KG-1 cells can be converted into DLC following stimulation with IL-4, GM-CSF, TNF- $\alpha$, IL-1 $\beta$, IL-6, and PGE 2 . Culture using the

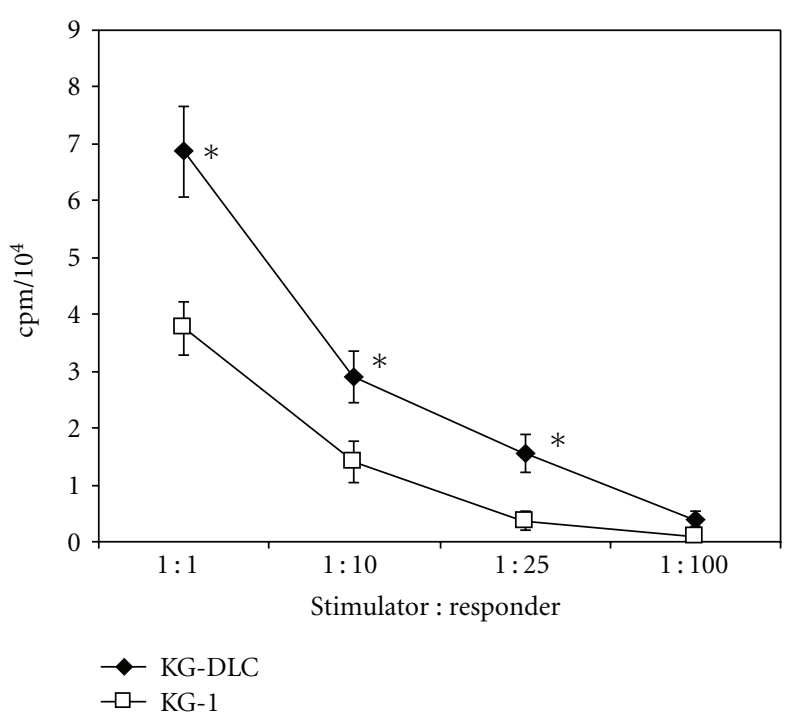

(a)

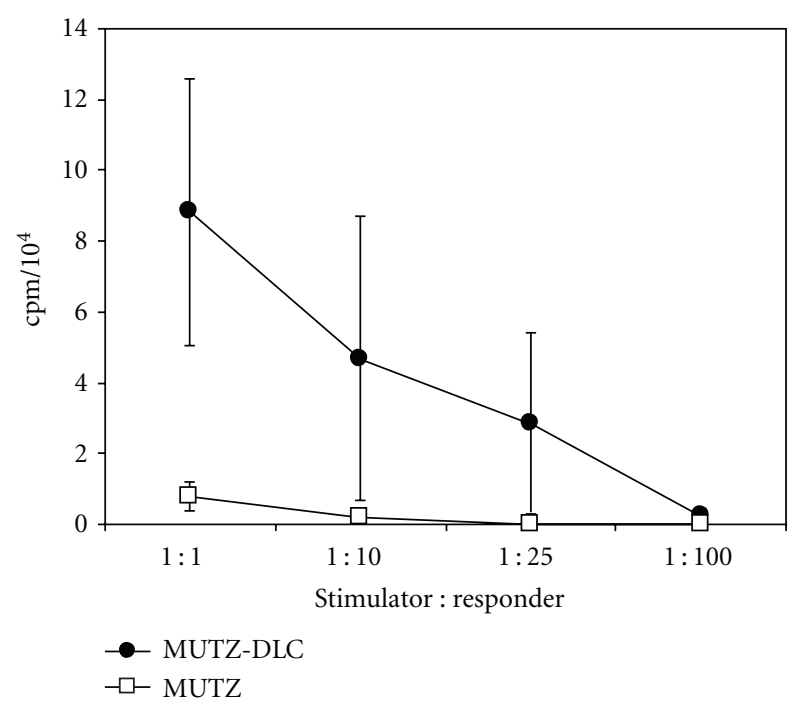

(b)

FIGURE 5: ${ }^{3} \mathrm{H}$-thymidine incorporation in MLC of DLC generated from KG-1 and MUTZ-3 cells. The MLC measurement was carried out after coculture with KG-1 (a) and MUTZ-3 (b) to check the donor PBMC proliferation. DLC were obtained from either KG-1 cells (KG-DLC) using FastDC culture or MUTZ-3 cells (MUTZDLC) using 7-day culture, and the proliferation stimulated by DLC was compared with that of normal KG-1 or MUTZ-3 cells. Results are expressed as mean \pm SEM, $n=3$ for using PBMC collected from three donors. ${ }^{*} P<0.05$ compared with KG-1.

FastDC method induced de novo expression of CD83 and up-regulation of CD86 and CD54. Failure to express CD1a is consistent with the findings of another report [10]. MUTZ3 DLC upregulated expression of CD54 and CD86 using the 7-day culture method but there was a slight increase in CD1a, CD40, CD80, and CD83. Our data suggest that the FastDC method is suitable for direct DC culture from PBMC. Both FastDC and the 7-day culture methods induced expression of CD1a, CD54, CD80, CD83, CD86, and HLA-DR. 


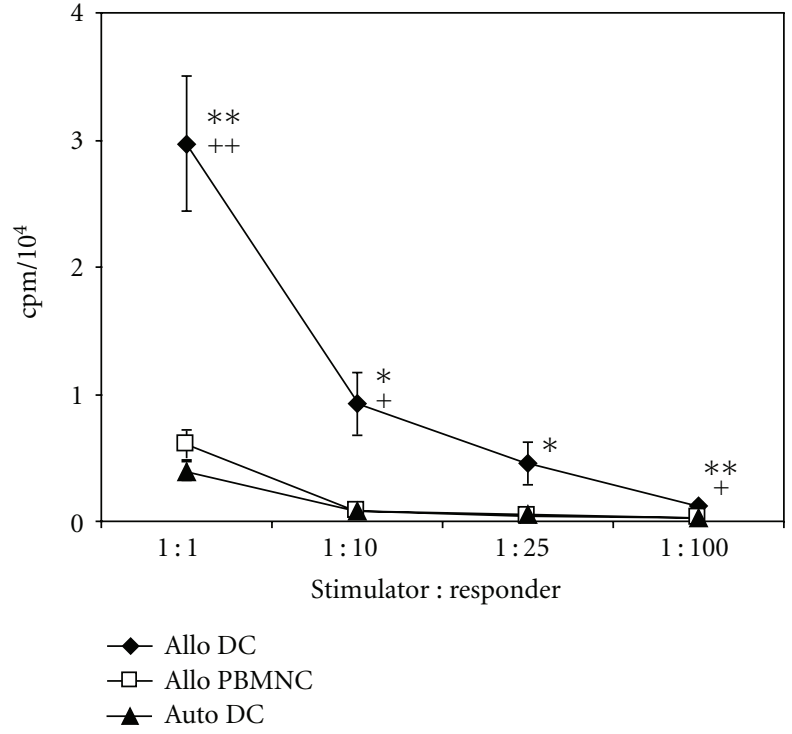

(a)

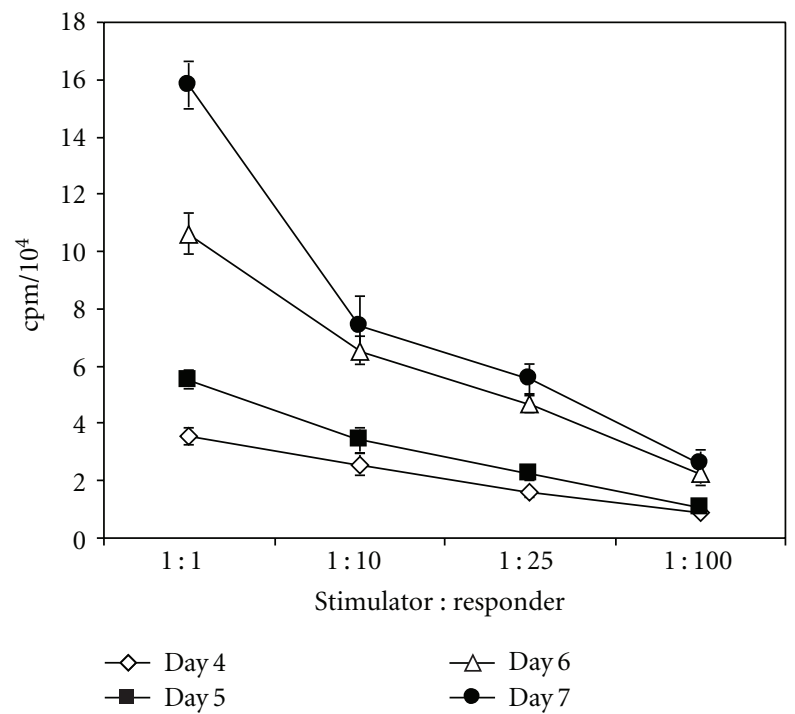

(b)

Figure 6: ${ }^{3} \mathrm{H}$-thymidine incorporation in MLCs using PBMCderived DC. MLC measurement was carried out after coculture for 4 days using DC generated from PBMC by FastDC (a). In the same experiment, allogeneic DC were compared with allogeneic PBMC for their stimulation ability, as well as with autologous DC. DC produced from FastDC culture were also used for an MLC time course study from day 4 to day 7 (b). Results are mean \pm SEM, $n=3$ for three tested samples. ${ }^{*} P<0.05,{ }^{* *} P<0.01$ compared with allogeneic $\mathrm{PBMC} ;{ }^{+} P<0.05,{ }^{++} P<0.01$ compared with autologous DC.

The expression of CD54, CD80, CD83, CD86, and HLA-DR was highest in 2-day cultures. In previous reports the FastDC method was only employed to culture DC from CD14 selected monocytes [17]. Our findings are in accordance with an earlier study by Teobald et al., in which upregulation of CD86 by activated KG- 1 cells was also greater than CD 83 and CD80 [18]. In contrast, another study showed that although
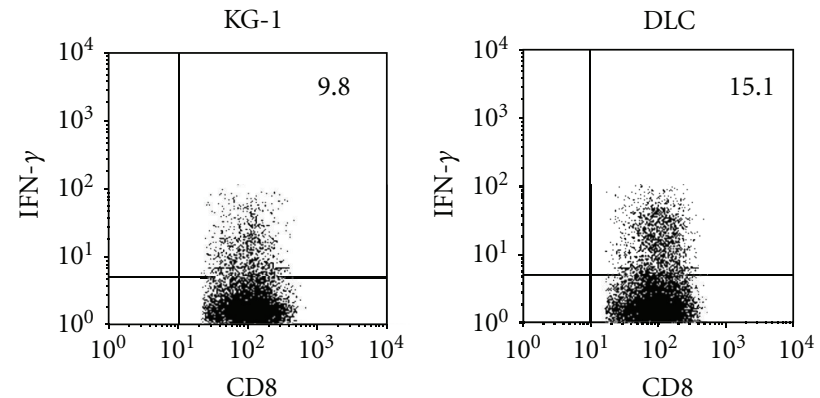

(a)
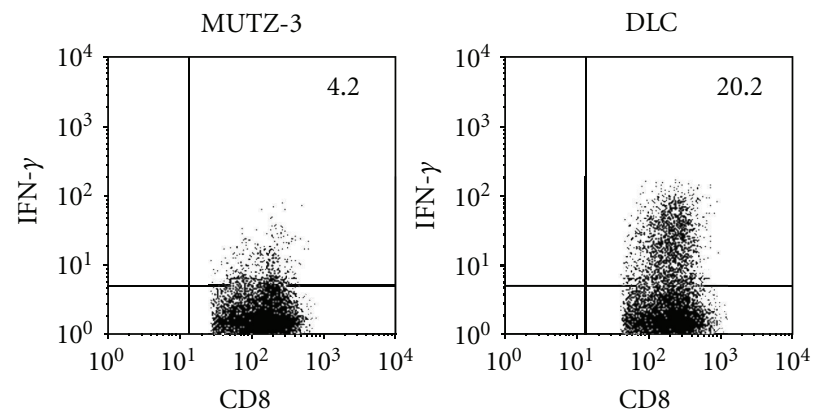

(b)
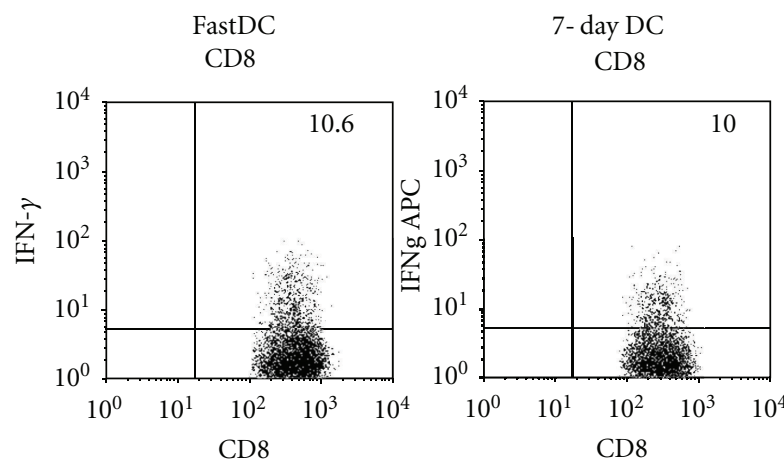

(c)

Figure 7: IFN- $\gamma$ production from $\mathrm{CD}^{+} \mathrm{T}$ cells. Irradiated KG-1 (a), MUTZ-3 (b) and the derived DLC as well as FastDC and 7-day culture DC derived from PBMC (c) were cocultured at the ration of $1: 10$ for 4 days with $5 \times 10^{5}$ responder PBMC loaded with CFSE. Before cells were harvested for IFN- $\gamma$ assay, $10 \mathrm{ng} / \mathrm{mL}$ PMA and $200 \mathrm{ng} / \mathrm{mL}$ ionomycin were added into the culture for the last $16 \mathrm{hrs}$ of incubation. Cells analysed were gated on CFSE positive $\mathrm{CD}^{+} \mathrm{T}$ cells. One representative result is shown.

CD80 and CD83 were not expressed on unstimulated KG-1 cells they were up-regulated following activation [10].

CD83 is known to be up-regulated amongst monocytederived DC and by MUTZ- 3 cells following culture using the conventional 7-day protocol [19]. Although our results are consistent with these findings, following the FastDC protocol MUTZ-3 DLC failed to express CD83 as well as CD80 and CD86. One possible explanation is that these "Fast DLC" may not be fully activated. We suggest that this is unlikely, given the fact that they were unable to endocytose FITC-labeled 
dextran, and they were capable of eliciting IFN $-\gamma$ production by naïve $\mathrm{T}$ cells.

In critical assays measuring DC function, we showed that DLC derived from both AML cell lines showed enhanced ability to stimulate proliferation in MLC. Moreover the production of IFN- $\gamma$ from KG-1 and MUTZ-3 DLC was significantly elevated when compared to control (unmodified) KG-1 and MUTZ-3 cells. Conventional DC generated using both FastDC and 7-day culture were able to phagocytose FITC-labeled dextran and stimulate HLA mismatched allogeneic responder cells better than unmodified PBMC stimulators. In previous in vitro functional studies, it was shown that FastDC derived from normal PBMC secrete IL12 (p70) when ligated with CD40 in the presence of IFN$\gamma$, induce antigen-specific T-cell proliferation and IFN- $\gamma$ production, and prime tumor antigen-specific cytotoxic Tcells $[3,15]$. Another immunotherapeutic approach using $e x$ vivo culture of AML cells to generate functional DC is of great interest; having the advantage that DLC generated from patient AML cells will present tumour antigens to donor $\mathrm{T}$ cells to stimulate CTL responses. In patient with acute leukaemia effective DC responses are often impaired and the absolute number of DC is reduced in children with acute lymphoblastic leukemia [20]. The tumor microenvironment may be inhibitory to growth of functional DC $[16,21]$; this may include defective T-cell activation as a result of a lack of crucial cell-cell signaling.

The signal pathways involved in DC activation are still not fully understood. Under pathological conditions the situation is more complex as the gene expression profiles and signaling pathways may be altered [22, 23]. In KG-1 cells, the protein kinase $\mathrm{C}(\mathrm{PKC})$ pathway is an important signal transduction route for DLC activation and inhibition of PKC may abort DLC induction [10]. Although Toll-like receptor 4 (TLR4) is expressed by MUTZ-3 cells the TLR pathway is impaired so LPS cannot stimulate the maturation of DLC differentiated from MUTZ-3 [24]. Other studies have shown that after culturing AML blasts for a short time with calcium ionophore DLC could be obtained which highly expressed the DC-related surface molecules such as CD80, CD83, CD86, and HLA-DR and which had the ability to promote CTL responses to blast cells $[7,25]$. These intracellular signal pathways control different DC functions such as surface antigen expression, cytokine secretion, and DC migration [26]. DC maturation after stimulation depends on the total outcome of the interactive network of signal transduction. Studies showed that human leukemic cell lines such as KG-1 might differentiate into DLC of mature phenotypes directly, bypassing the immature phase $[10,11]$. This direct maturation of KG-1 after culture with PMA and ionomycin not only increases the expression of CD83 as a maturation surface marker, but decreases the ability of endocytosis [18]. In our study, DLC differentiated from KG-1 or MUTZ-3 cells did not show improved endocytosis, and uptake level of Dextran-FITC was less than undifferentiated KG-1 or MUTZ-3 (data not shown). Previous reports showed that DLC generated from MUTZ-3 include two stages: culture with GM-CSF, IL-4, and low dose of TNF- $\alpha$ to obtain immature DLC and further high-dose TNF- $\alpha$ treatment to induce the maturation [17]. In this study, we observed some increase in the levels of DC-related surface markers after maturation in 7-day culture.

We conclude that phenotypic DLC can be derived from the AML cell lines KG-1 and MUTZ-3 and that these induce CTL responses. Moreover, FastDC culture could be utilised to generate DC from $\mathrm{PBMC}$ as well as $\mathrm{CD} 14^{+}$selected cells and these were capable of promoting allogeneic PBMC proliferation up to day 7 . These findings will be of great importance in determining the choice of cells and culture conditions for the development of future immunotherapeutic strategies to combat AML and other cancers.

\section{Acknowledgments}

The authors greatly appreciate the valuable suggestions from Dr. Russell Garland and the support from donors and staff of Apheresis Unit, National Blood Service in Bristol. This paper is supported by a program grant from the NHS Blood and Transplant.

\section{References}

[1] A. Blair, N. J. Goulden, N. A. Libri, A. Oakhill, and D. H. Pamphilon, "Immunotherapeutic strategies in acute lymphoblastic leukaemia relapsing after stem cell transplantation," Blood Reviews, vol. 19, no. 6, pp. 289-300, 2005.

[2] G. M. Zou, J. Martinson, W. Y. Hu, Y. Tam, and H. G. Klingemann, "The effect of LIGHT in inducing maturation of monocyte-derived dendritic cells from MDS patients," Cancer Immunology, Immunotherapy, vol. 53, no. 8, pp. 681-689, 2004.

[3] M. Dauer, B. Obermaier, J. Herten et al., "Mature dendritic cells derived from human monocytes within 48 hours: a novel strategy for dendritic cell differentiation from blood precursors," Journal of Immunology, vol. 170, no. 8, pp. 40694076, 2003.

[4] A. Blair, A. W. Rowbottom, S. J. Browne et al., "An optimised biphasic culture system for the generation of functional dendritic cells from patients with acute lymphoblastic leukaemia at presentation and in clinical remission," Leukemia, vol. 15, no. 10, pp. 1596-1603, 2001.

[5] B. D. Harrison, J. A. Adams, M. Briggs, M. L. Brereton, and J. A. Yin, "Stimulation of autologous proliferative and cytotoxic T-cell responses by "leukemic dendritic cells" derived from blast cells in acute myeloid leukemia," Blood, vol. 97, no. 9, pp. 2764-2771, 2001.

[6] Y. Osman, M. Takahashi, Z. Zheng et al., "Activation of autologous or HLA-identical sibling cytotoxic T lymphocytes by blood derived dendritic cells pulsed with tumor cell extracts," Oncology Reports, vol. 6, no. 5, pp. 1057-1063, 1999.

[7] T. M. Westers, A. G. M. Stam, R. J. Scheper et al., "Rapid generation of antigen-presenting cells from leukaemic blasts in acute myeloid leukaemia," Cancer Immunology, Immunotherapy, vol. 52, no. 1, pp. 17-27, 2003.

[8] S. J. A. M. Santegoets, A. J. M. van den Eertwegh, A. A. van de Loosdrecht, R. J. Scheper, and T. D. de Gruijl, "Human dendritic cell line models for DC differentiation and clinical DC vaccination studies," Journal of Leukocyte Biology, vol. 84, no. 6, pp. 1364-1373, 2008. 
[9] A. L. Ackerman and P. Cresswell, "Regulation of MHC class I transport in human dendritic cells and the dendritic-like cell line KG-1," Journal of Immunology, vol. 170, no. 8, pp. 41784188, 2003.

[10] D. C. St. Louis, J. B. Woodcock, G. Franzoso et al., "Evidence for distinct intracellular signaling pathways in CD34+ progenitor to dendritic cell differentiation from a human cell line model," Journal of Immunology, vol. 162, pp. 3237-3248, 1999.

[11] C. Berges, C. Naujokat, S. Tinapp et al., "A cell line model for the differentiation of human dendritic cells," Biochemical and Biophysical Research Communications, vol. 333, no. 3, pp. 896907, 2005.

[12] P. Azam, J. L. Peiffer, D. Chamousset et al., "The cytokinedependent MUTZ-3 cell line as an in vitro model for the screening of contact sensitizers," Toxicology and Applied Pharmacology, vol. 212, no. 1, pp. 14-23, 2006.

[13] H. J. Bontkes, J. J. Ruizendaal, D. Kramer et al., "Constitutively active STAT5b induces cytokine-independent growth of the acute myeloid leukemia-derived MUTZ-3 cell line and accelerates its differentiation into mature dendritic cells," Journal of Immunotherapy, vol. 29, no. 2, pp. 188-200, 2006.

[14] H. Quentmeier, M. Zaborski, and H. G. Drexler, "The human bladder carcinoma cell line 5637 constitutively secretes functional cytokines," Leukemia Research, vol. 21, no. 4, pp. 343-350, 1997.

[15] M. Dauer, K. Schad, J. Herten et al., "FastDC derived from human monocytes within $48 \mathrm{~h}$ effectively prime tumor antigen-specific cytotoxic T cells," Journal of Immunological Methods, vol. 302, no. 1-2, pp. 145-155, 2005.

[16] J. Rasaiyaah, K. Yong, D. R. Katz, P. Kellam, and B. M. Chain, "Dendritic cells and myeloid leukaemias: plasticity and commitment in cell differentiation," British Journal of Haematology, vol. 138, no. 3, pp. 281-290, 2007.

[17] A. J. Masterson, C. C. Sombroek, T. D. De Gruijl et al., "MUTZ-3, a human cell line model for the cytokine-induced differentiation of dendritic cells from CD34+ precursors," Blood, vol. 100, no. 2, pp. 701-703, 2002.

[18] I. Teobald, D. J. Dunnion, M. Whitbread, S. J. Curnow, and M. J. Browning, "Phenotypic and functional differentiation of KG-1 into dendritic-like cells," Immunobiology, vol. 213, no. 1, pp. 75-86, 2008.

[19] S. J. A. M. Santegoets, M. W. J. Schreurs, A. J. Masterson et al., "In vitro priming of tumor-specific cytotoxic T lymphocytes using allogeneic dendritic cells derived from the human MUTZ-3 cell line," Cancer Immunology, Immunotherapy, vol. 55, no. 12, pp. 1480-1490, 2006.

[20] B. Maecker, D. Mougiakakos, M. Zimmermann et al., "Dendritic cell deficiencies in pediatric acute lymphoblastic leukemia patients," Leukemia, vol. 20, no. 4, pp. 645-649, 2006.

[21] A. G. S. Buggins, D. Milojkovic, M. J. Arno et al., "Microenvironment produced by acute myeloid leukemia cells prevents $\mathrm{T}$ cell activation and proliferation by inhibition of NF-kappaB, c-Myc, and pRb pathways," Journal of Immunology, vol. 167, no. 10, pp. 6021-6030, 2001.

[22] H. Bruchova, M. Kalinova, and R. Brdicka, "Array-based analysis of gene expression in childhood acute lymphoblastic leukemia," Leukemia Research, vol. 28, no. 1, pp. 1-7, 2004.

[23] E. Sakhinia, M. Faranghpour, J. A. L. Yin, G. Brady, J. A. Hoyland, and R. J. Byers, "Routine expression profiling of microarray gene signatures in acute leukaemia by real-time PCR of human bone marrow," British Journal of Haematology, vol. 130, no. 2, pp. 233-248, 2005.
[24] D. K. Kwang, S. C. Choi, Y. W. Noh et al., "Impaired responses of leukemic dendritic cells derived from a human myeloid cell line to LPS stimulation," Experimental and Molecular Medicine, vol. 38, no. 1, pp. 72-84, 2006.

[25] M. Waclavicek, A. Berer, L. Oehler et al., "Calcium ionophore: a single reagent for the differentiation of primary human acute myelogenous leukaemia cells towards dendritic cells," British Journal of Haematology, vol. 114, no. 2, pp. 466-473, 2001.

[26] T. Luft, E. Rodionova, E. Maraskovsky et al., "Adaptive functional differentiation of dendritic cells: integrating the network of extra- and intracellular signals," Blood, vol. 107, no. 12, pp. 4763-4769, 2006. 


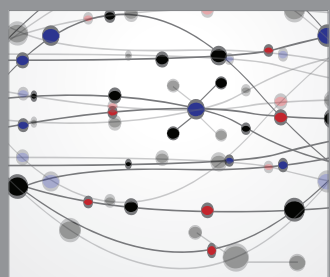

The Scientific World Journal
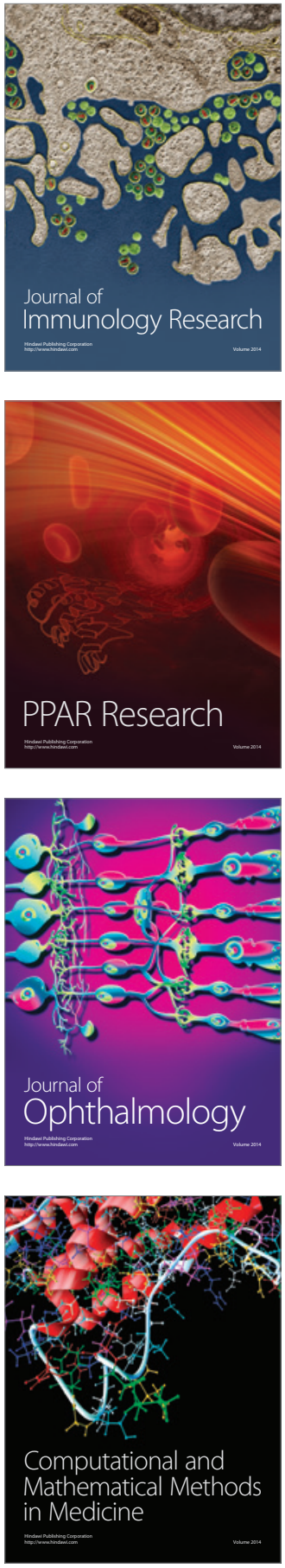

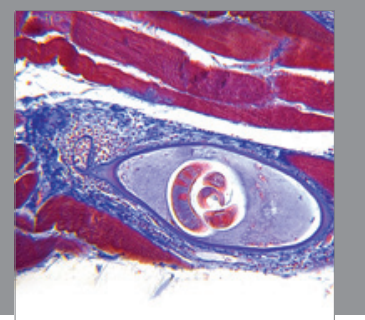

Gastroenterology

Research and Practice
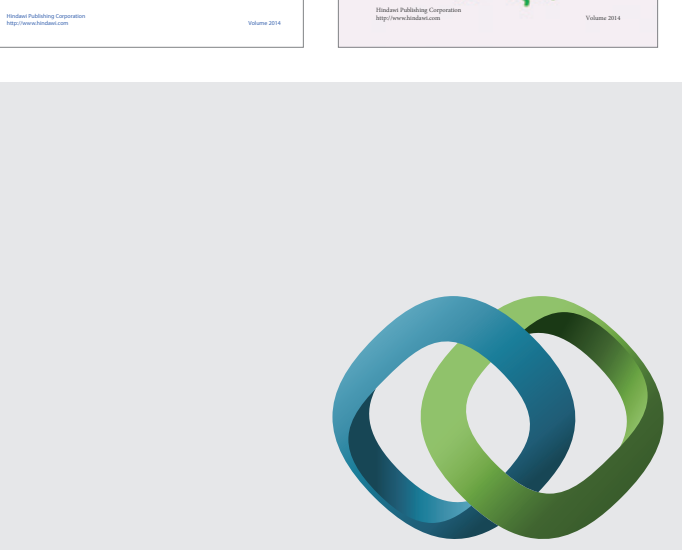

\section{Hindawi}

Submit your manuscripts at

http://www.hindawi.com
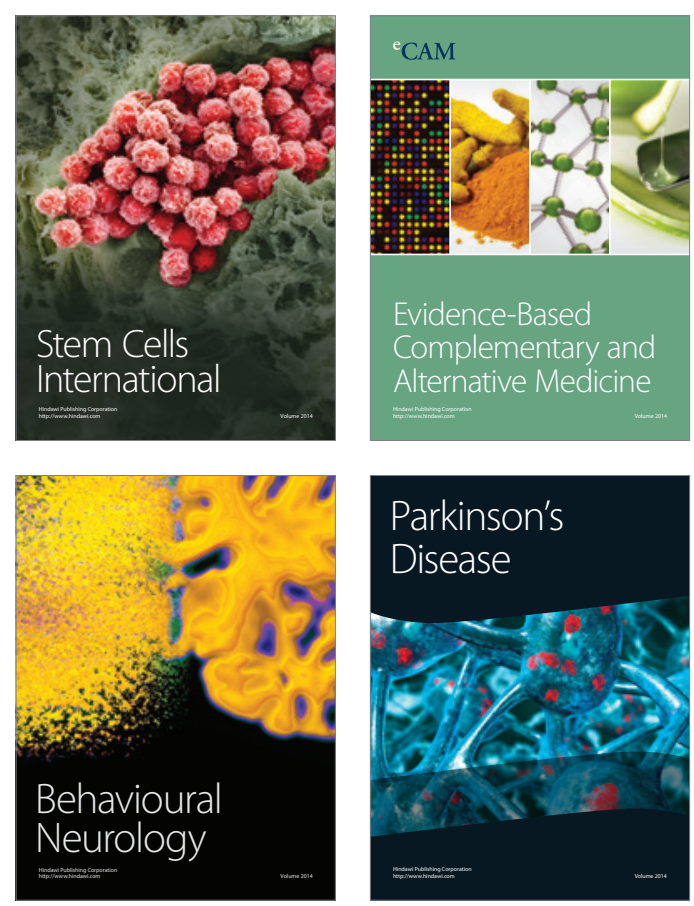

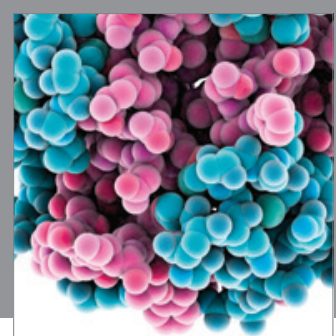

Journal of
Diabetes Research

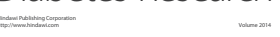

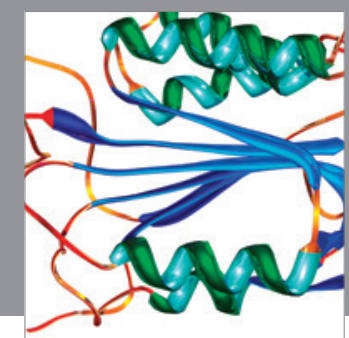

Disease Markers
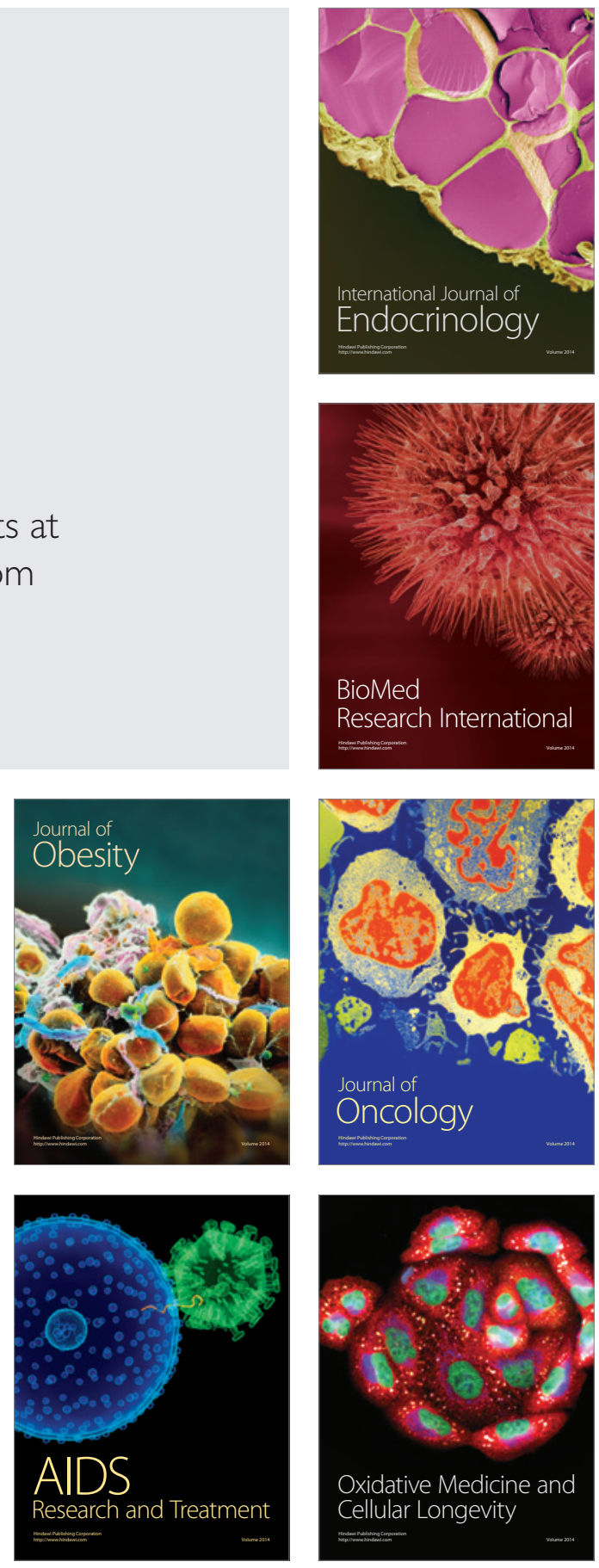